\title{
Invisible Medical Image Watermarking using Edge Detection And Discrete Wavelet Transform Coefficients
}

\author{
Ramanand singh, Piyush Shukla, Paresh Rawat, Prashant Kumar Shukla
}

\begin{abstract}
Protection and authentication of medical images is essential for the patient's disease identification and diagnosis. The watermark in medical imaging application needs to be invisible and it is also required to preserve the low and high frequency features of image data which makes watermarking a difficult assignment. Within this manuscript an unseen medical image watermarking approach is projected apply edge detection in the discrete wavelet transform domain. The wavelet transform is brought into play to decay the medical picture interested in multi-frequency secondary band coefficients. The edge detection applies to high frequency wavelet group in the direction of generating the boundary coefficients used as a key. The Gaussian noise pattern is utilized as watermark as well as embedded within the edge coefficients around the edges. To add the robustness scaled dilated edge coefficient is added with the edge coefficients to generate the watermarked image. Preserving the small frequency secondary band fulfills the information requirement of the medical imaging application. At the same time as adding together the watermark during high frequency sub-bands improve the watermark invisibility. To add additional robustness the dilation is applied on the edged coefficient before being embedded with sub band coefficients. presentation of the technique is experienced on the dissimilar set of medical imagery as well as evaluation of the proposed watermarking method founds it robust not in favor of the different attacks such at the same time as filtering, turning round plus resizing. Parametric study foundation going on Mean Square Error along with Signal to Noise Ratio shows that how good method performs for invisibility.
\end{abstract}

Keywords- Medical Images, Watermarking, Discrete Wavelet Trasform, Edge Detection, Dilation, Invisibility.

\section{INTRODUCTION}

The watermarking skill creates exercise of the information with the intention of the human being watch has merely a partial ability to watch differences. Small modifications in the dye value of a picture are subconsciously accurate through the eye, so that the viewer does not observe any variation. In several medical applications dissimilar variety of medical imagery is exercise for diagnosis otherwise recognition of the illness in the human beings. Consequently protected the medical imaging information is a necessary task.

Revised Manuscript Received on November 06, 2019.

Ramanand singh, Department of Electronics \& Communication Rabindranath Tagore University, Bhopal

Dr. Piyush Shukla,UIT RGPV Bhopal

Dr. Paresh Rawat, Deptt. Of EC, Sistech, Bhopal, India

Dr Prashant Kumar Shukla,Deptt. of CSE, SOET, Jagran Lakecity Univercity, Bhopal ${ }^{4}$
The medical imaging data are shared exponentially on the local or public networks all around the globe as digital images or videos data [3]. Since captures MRI image and CT scan images or X-ray images may look like same for two different patients, therefore, there is always a probability of imaging reports interchange. Therefore generates the needs of copyright protection and authenticating the medical images before being diagnosed [13]. The authentication is done through watermarking techniques. The major watermarking challenges include avoiding the effect of different kind of attacks such as; Noise, cropping, and compression attacks [9]. Edge detection has been used by many researchers for embedding the watermark in digital images robustly. Edges caries the strong high frequency information content as features of the images.

Table 1 Requirements of medical image

\begin{tabular}{|l|ll|}
\multicolumn{2}{c}{ Watermarking } \\
\hline \multicolumn{1}{|c|}{ Type } & & Requirements \\
\hline Compulsory & $\bullet$ & Invisibility \\
& $\bullet$ & Information \\
& & preservation \\
& $\bullet$ & Security (protection) \\
& $\bullet$ & Low noise sensitivity \\
\hline Desirable & $\bullet$ & Reconstruction \\
& $\bullet$ & Robustness \\
& & \\
\hline
\end{tabular}

Thus Medical images may have sensitive information's on the edges. On the other hand it is also true that the edges will be customized during the compression process if high compression ratios are required. Therefore, it is required to analyze the success edge based watermarking under compression attacks. The major requirements of the medical image watermarking can be classified into the mandatory and desirable requirements and are tabulated in Table 1 . It can be observed that the invisibility is the major requirement of the medical imaging data on the web or local networks, and higher reconstruction efficiency strictly is desirable under various attacks. The paper addresses the limitations of existing edge detection based watermarking methods designed for the data authentication as discussed in section 2 . The wavelet based watermarking methods and the concepts of wavelet transform are briefly described in the next section. The proposed watermarking methodology using the wavelet based edge detection along with the algorithm is explained. The result presents the comparison of various edge detection methods using the wavelet transform followed by the results of the proposed watermarking method. 


\section{Invisible Medical Image Watermarking using Edge Detection And Discrete Wavelet Transform Coefficients}

The performance is evaluated using the quality measures, and finally conclusions are specified.

\section{PREVIOUS WORK}

The edge detection based watermarking methods are broadly classified as shown in Figure 1.

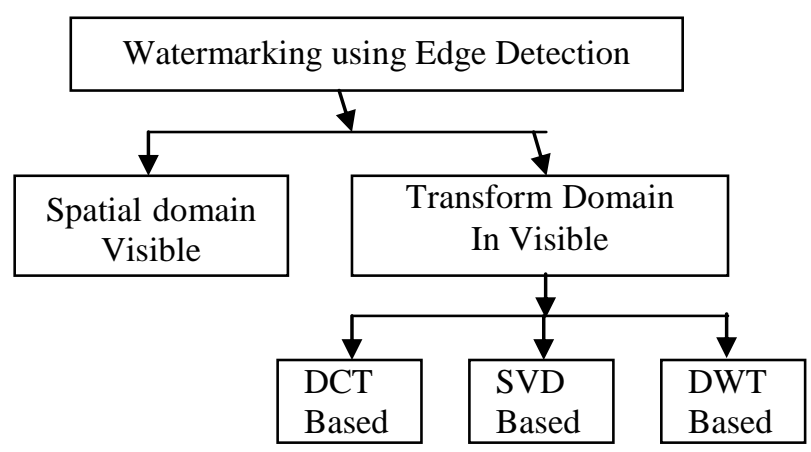

Figure 1 Classification of Edge based watermarking

Invisible watermarking is popular in transform domain based methods and suitable for the medical image watermarking [13, 18]. The invisible watermarking is widely used in copyright, security scheme, which is projected to prevent otherwise detect illegal copying of digital images. Various watermarking algorithms were proposed in the past to create robust medical image watermarking. The wavelet transform has been widely used for watermark insertion since long back [1, 2, 5, and 8]. Xia, et al. [19] projected a watermarking system foundation going on the Discrete Wavelet Transform (DWT). The watermark, represent the same as Gaussian noise, was additional in the direction of the center as well as high frequency bands of the picture. Sections of the watermark were taken out and associated by way of part of the unique watermark. Tao and Eskicioglu [20] have presented a wavelet based watermark embedding technique. They have proposed to embed the binary symbol as watermark using every the four wavelet sub band. Other than they have used different scaling factors in each sub band for embedding the watermarks. But this method is not so suitable for medical image authenticating since the probability of large information change is there.

A. Edge based methods: Edge detection is most popular for image watermarking techniques, because an edge in a medical image represents the features or contours where major significant changes occur in an image. Gradient vector having a certain magnitude and direction and can be used to perform basic edge detection and magnitude and phase are mathematically represented as.

$$
\begin{gathered}
\nabla f=\left[\begin{array}{l}
\frac{\partial f}{\partial x} \\
\frac{\partial f}{\partial y}
\end{array}\right]=\sqrt{M_{x}^{2}+M_{y}{ }^{2}} \\
\approx\left|M_{x}\right|+\left|M_{y}\right| \\
\operatorname{dir}(\nabla f)=\tan ^{-1}\left(M_{x} / M_{y}\right)
\end{gathered}
$$

Where $M$ gradients can be estimated using different edge detection masks with finite difference method [5]. Estimating the gradient with finite differences can be given by

$$
\begin{array}{ll}
=\lim _{h \rightarrow 0} \frac{f(x+h, y)-f(x, y)}{h} & \frac{\delta f}{\delta x} \\
=\lim _{h \rightarrow 0} \frac{f(x, y+h)-f(x, y)}{h} & \frac{\delta f}{\delta y}
\end{array}
$$

Commonly used as edge detector for the image watermarking is the Sobel Edge mask. This mask or filters are the average operator which is more likely to a Gaussian; this makes it better for removing some white noise.

X Gradiant $\quad\left[\begin{array}{rrr}-1 & -2 & -1 \\ 0 & 0 & 0 \\ 1 & 2 & 1\end{array}\right] \quad\left[\begin{array}{rrr}-1 & 0 & 1 \\ -2 & 0 & 2 \\ -1 & 0 & 1\end{array}\right]$

$\mathbf{F x}$

$\mathbf{F}_{\mathbf{y}}$

This can be observed clearly from Figure 2 an example of the basic edge detection using Sobel mask.
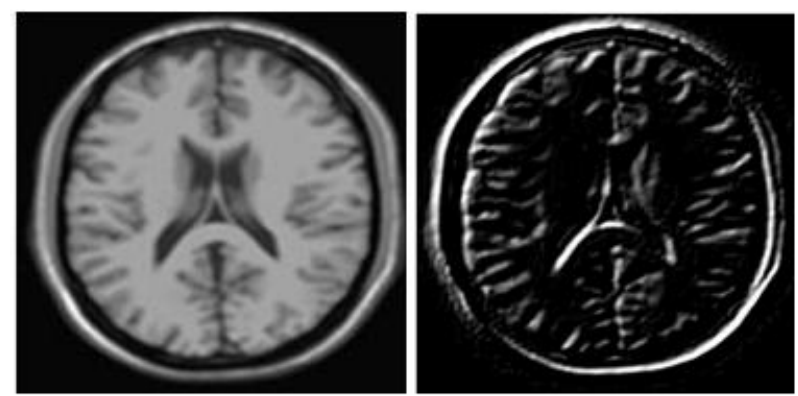

Figure 2 Edge detection examples for CT scan image

B. Spatial domain Watermarking: In this category the watermark is set using the pixel level information of the picture. There are many spatial domain methods $[8,19]$ which are designed for watermarking the images. Usually temper detection based methods are common in spatial domain techniques. J. M. Zain et al. [8] Have proposed to use the tamper detection and recovery based method for medical image watermarking. These are the estimation based methods. S. C. Liew et al. [9] Has proposed a reversible interfere detection as well as revival based medical picture watermarking method was a spatial domain method. Vikas Tyagi [16] has used least significant bit based cryptography method of data hiding.

C. DCT based Watermarking: Saeed et al. [7] Projected. An algorithm foundation going on combined DWT-DCT Transformation. A double watermarked symbol is twisted through Arnold cat chart as well as set in in the convincing coefficient set of a 3 stage DWT transformed of a crowd picture.

Subsequently change of the entire DCT chosen DWT subband be computed at the same time as the PN series of the watermark bit be set in within the center frequency coefficients of the consequent DCT building block. Although the technique appears just before be real greatly compound which requirements better occasion plus dispensation authority. 
D. Wavelet based Watermarking: John et al. [3] Have used wavelet edge detection along with the contrast sensitivity function for embedding the watermark this method was modified by their next work. John et al. [1] Have proposed to use the wavelet based edge detection for embedding the watermark. The Gaussian noise is used as watermark template. They have first time used morphological dilation for adding the robustness to the system.

Yingl et al. [2] Have designed the watermarking method using texture block in DWT domain and the canny edge detector mask. The method was useful to hide text within the image. A new shift invariant edge detection mask is proposed to us by Apeksha et al. [5]. They have modified the method proposed by John et al. [3] They have proved that the shift invariant mask can provide better invisibility.

Narong et al. [4] Have used the blue color gap of the host picture for embedding the watermark using the DWT coefficients based watermarking. The method seems good for robust watermarking of the patient identifications where the text image is embedded in patient MRI data image. Li et al (2003) utilizes a dual chart toward predetermine the edges of a picture in their picture validation system. A different procedure has been projected to decrease the computational difficulty plus to get better the precision of the movement opinion.

Why using wavelet Edge Detectors Wavelet study is a local study; it is mainly appropriate intended for timefrequency study [2], which is necessary intended for singularity recognition. This was a main incentive intended for the revision of the wavelet transform in arithmetic as well as in practical area. Using wavelet transform improves the robustness and invisibility of the watermark. The wavelet transform describe the limited reliability of imagery by decaying them into basic coefficients so as to be well limited to a small area in gap and frequency together. This not just give details the fundamental method of traditional edge detectors, other than as well point to a method of build most advantageous edge detectors below exact operational circumstances the same as in multi decision.

E Medical image watermarking: lot of methods are developed for medical image watermarking earlier, Ni et al [6] have developed a reversible medical image watermarking method using location gapes and histogram shifting methods. They have developed a new method for bit map location recording. But method may lose medical data sometimes. Smita et al. [10] Have used integer to integer wavelet transform for generating reversible medical image watermarking. Method embedded the watermark in the maximum entropy block. Sometimes for specific medical images this may lead to edge variations of patient image. Therefore the major concern with medical image watermarking is that the image content must not be lost. Thus watermark must be robust to attacks.

\section{WAVELET BASED WATERMARKING}

Discrete wavelet transform (DWT) are widely utilized for the watermarking algorithms. Since wavelets conveniently break the image into the multiple approximation and details, thus are widely used in watermarking applications. The DWT based wavelets transform efficiently isolates and manipulate the high frequency along with low frequency sub bands so that watermark may be easily embedded within the sub band which is less sensitive to the human vision.
Wavelets are get beginning a solitary example wavelet $\psi(t)$ name mother wavelet by scaling as well as shifting.

$$
=\frac{1}{\sqrt{a}} \psi\left(\frac{t-b}{a}\right) \quad \psi_{a, b}(t)
$$

Where, $a$ is the scaling factor and $b$ is the shifting factor intended for corresponding in oorder fromwithin a video, the straight move toward can typically be complete to work [11]. Let $\mathrm{I}_{0}$ be a grayscale image decomposed by wavelet, then the first level decomposition will be:

$$
I_{H L 1}+I_{H H 1 \mathrm{I}} \quad I_{0}=I_{L L 1}+I_{L H 1}+
$$

Where, the $\mathrm{I}_{\mathrm{LL} 1}$ represents the low frequency parts and $\mathrm{I}_{\mathrm{LHI}}$. $\mathrm{I}_{\mathrm{HLI}} \mathrm{I}_{\mathrm{HHI}}$ represents high frequency sub-bands details The Figure 3 demonstrates the pattern of a 2-dimensional, two level discrete wavelet decay for CT image.

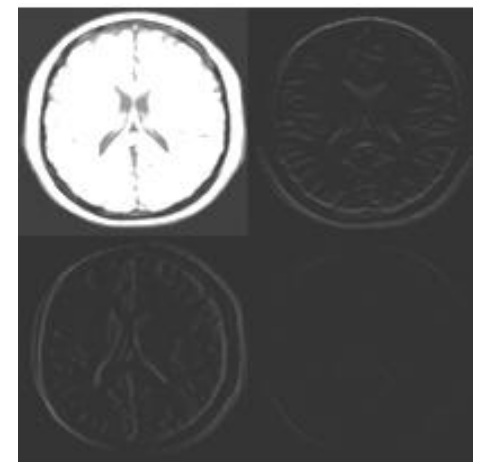

Figure 3 DWT decomposition of CT scans image a) First level, b) Second Level

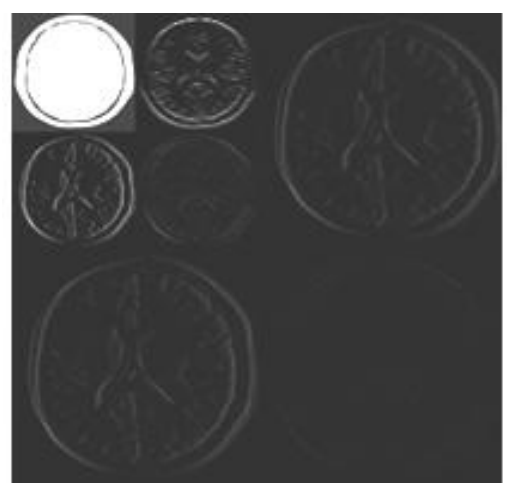

It can be observed from the Figure that the high frequency sub band of the Figure $3 \mathrm{~b}$ ) that it is almost having very few features thus provide more invisibility if used for watermarking.

\section{WAVELET EDGE DETECTION}

In wavelet based edge detection, first medical images are decomposed using the discrete wavelet transform using basic Haar wavelet. Then the edge of wavelet coefficient is calculated for the desired sub band as shown in LL band in Figure 4. And then finally Inverse Wavelet transformation is taken. 
Invisible Medical Image Watermarking using Edge Detection And Discrete Wavelet Transform Coefficients
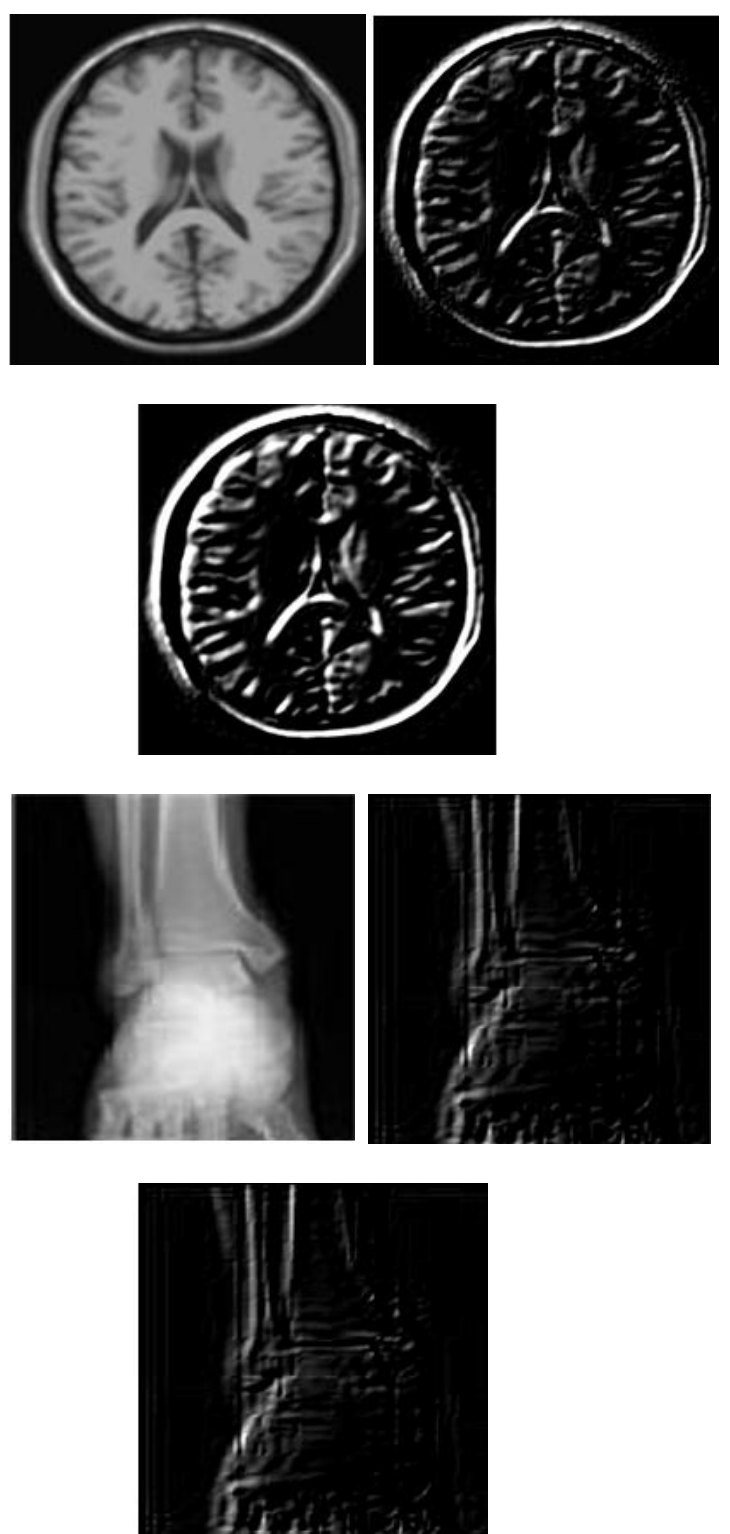

Figure 4 Wavelet edge detection comparison a) unique image b) basic edge detection c) wavelet based edge detection

\section{MODIFIED DWT BASED WATERMARKING}

It can be observed from Figure 4 that wavelet based edge detection gives more features. But it also needs the more coefficients scaling for the better invisibility and to avoid the noise effects. This was a main incentive intended for the learned of the wavelet transform in arithmetic as well as in useful domains. Using wavelet transform improves the robustness and invisibility of the watermark.

In the current paper a modified watermarking rule is proposed by using scaling the coefficients. The existing watermark rule [5] was

$$
\mathrm{W}(\mathrm{x}, \mathrm{y})=(1-\alpha) *\left(\mathrm{Y}_{\mathrm{x}, \mathrm{y}}^{\mathrm{L}}-\mathrm{X}_{\mathrm{x}, \mathrm{y}}^{\mathrm{L}}\right)+
$$

$\alpha N_{x, y}$

First modification is to use the Sobel mask instead of shift invariant mask. Furthermore, the addition might be scaled according in the direction of the decay level along with the edge set to which the coefficients are in the right place too. Modified rule for watermarked picture is reached by avoiding the more scaling of the noise template. This improves the computational efficiency and since scaling the uniform for edge and nice template.

$$
\left.\left.\mathrm{X}_{\mathrm{x}, \mathrm{y}}^{\mathrm{L}}\right)+N_{x, y}\right)
$$

$$
\mathrm{W}(\mathrm{x}, \mathrm{y})=(1-\alpha) *\left(\left(\mathrm{Y}_{\mathrm{x}, \mathrm{y}}^{\mathrm{L}}-\right.\right.
$$

In the projected watermarking process the input true color picture is primarily converted to gray images for reducing the computation overload. Then the proposed wavelet based edge detection first second level DWT is performed and then edge detection of the $\mathrm{HH}$ component $\mathrm{X}_{\mathrm{x}, \mathrm{y}}^{\mathrm{L}}$ is generated. Sobel mask is preferred here for the edge detection since it gives brighter features. The dilation of the edge coefficient generates $\mathrm{Y}_{\mathrm{x}, \mathrm{y}}^{\mathrm{L}}$ Coefficients. And the scaled difference of these coefficients is used along with the added Gaussian noise template to generate the watermark image.. Finally, using the inverse DWT the watermarked output is generated as shown in Figure 5.

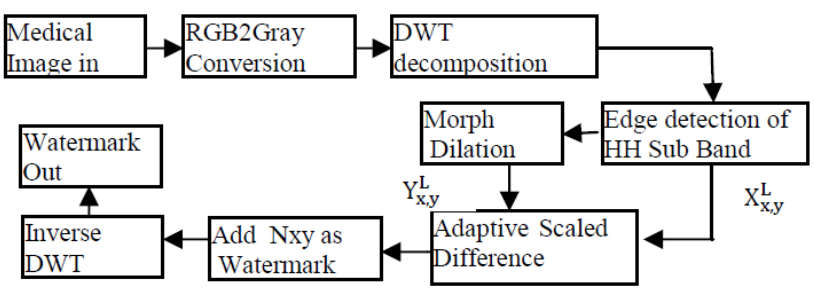

Figure 5 Proposed Watermarking Schemes

a) Flow Chart of the Algorithm The sequential flow of the watermark insertion algorithm is shown in Figure 6.

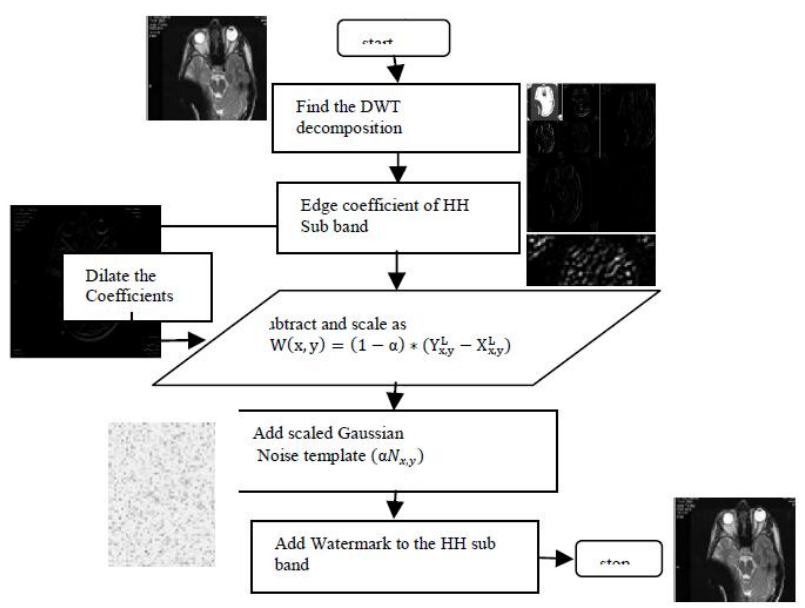

Figure 6 Flowchart of Watermark insertion process

\section{RESULTS AND DISCUSSIONS}

In this manuscript the edge detected high frequency discrete wavelet transform (DWT) coefficients are used for medical image watermarking. This part of paper presents various tentative outcome of our effort on top of projected digital picture watermarking process have obtaining. The different edge detection process is put into practice. The paper also presents the performance of the watermarking method under various kinds of filtering attacks.

\section{a) Result of the Classical Edge detectors with DWT}

The results of the edge detection with wavelet transform for MRI medical image are shown in Figure 7. Since MRI image contains very precise information, thus we need an edge mask having more information. Sobel mask is used here.

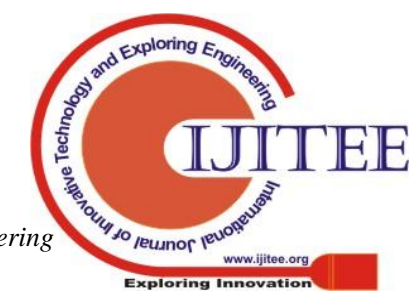




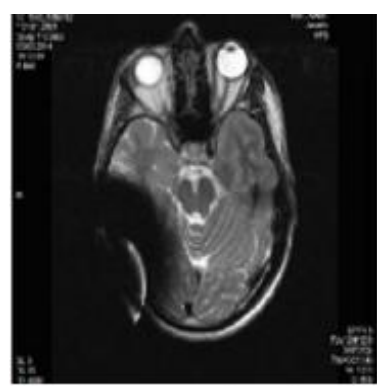

a) Unique picture
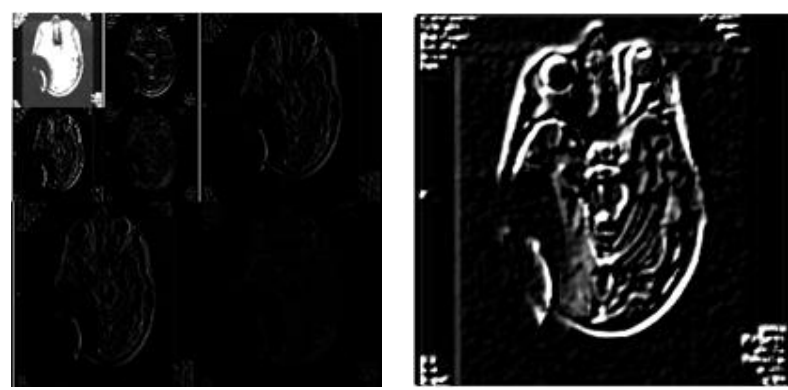

c) Second level DWT picture picture

Figure 7 Wavelet edge detection for MRI patient _image_1

It can be observed from the Figure 7 that Sobel edge detector mask do improved than additional edge masks in conditions of sharpness along with the brightness of the edges and it also contains more precise features too.

b) Sequential Result of the projected Watermarking process with DWT

Comparisons of the sequential watermark insertion process stage wise according to the flow chart are shown in Figure

8. Results for Sobel edge mask and dilation mask size of $2 \mathrm{x}$ 2 are presented with our proposed scaled dilation method. It is able to be experiential that our projected technique does smoother than other technique thus improves invisibility of watermark. For comparison dilation is calculated by scaling parameter $\alpha=0.8$ for invisibility.
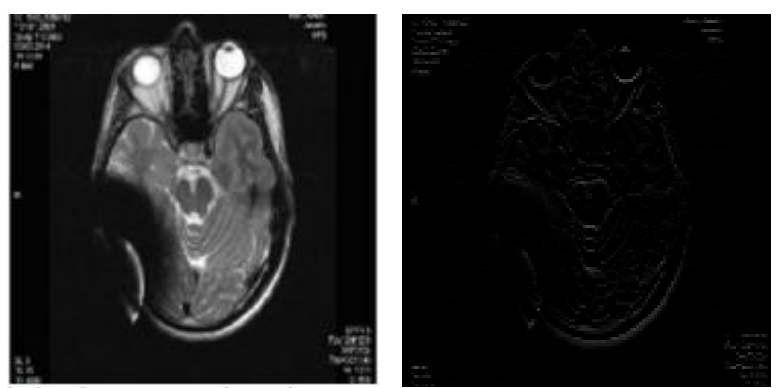

Origina MRI image d) Wavelet HH coeficient
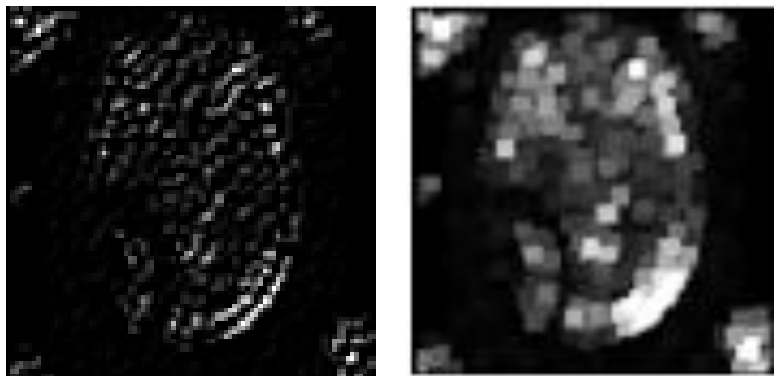

Original edge image d) Dilated edge coefficients
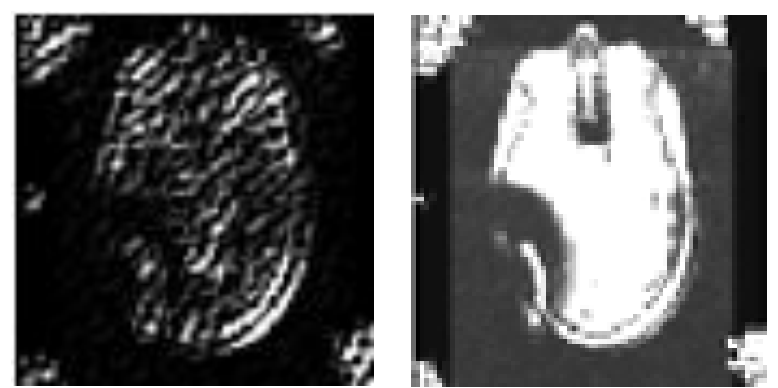

e) Difference image f) Watermark with our scaling method at level 1

Figure 8 Sequential results stage wise for MIR image

The comparison of the results of watermarked image for the results of shift invariant mask of Apeksh et al [5] and our proposed method are given inside the Figure 9 It can be observe that the projected technique minimizes the error difference after watermarking and gives better invisibility. Also the comparison of the MSE and SNR are presented in the Table 2 and 3 as shown below, and found to improve by proposing watermark rule. it preserve be experiential so as to the projected technique act upon improved for most of the medical image modalities. Except for CT image in comparison and SNR is more with our method.

Table 2 MSE comparison

\begin{tabular}{|l|l|l|l|}
\hline Method & $\begin{array}{l}\text { MRI } \\
\text { image }\end{array}$ & $\begin{array}{l}\text { CT } \\
\text { image }\end{array}$ & $\begin{array}{l}\text { X-ray } \\
\text { image }\end{array}$ \\
\hline $\begin{array}{l}\text { Apeksh } \\
\text { Et Al. [5] }\end{array}$ & $\mathbf{2 1 . 7 8 0 8}$ & $\mathbf{4 . 9 7 0}$ & $\mathbf{3 . 6 9 5 8}$ \\
\hline Proposed & $\mathbf{1 3 . 7 4 0 9}$ & $\mathbf{1 3 . 7 0}$ & $\mathbf{5 . 7 1 0 3}$ \\
\hline
\end{tabular}

\begin{tabular}{|l|l|l|l|}
\hline Method & MRI & CT & X-ray \\
& image & image & image \\
\hline Apeksh & $\mathbf{3 . 0 6 8}$ & $\mathbf{9 . 2 0 9}$ & $\mathbf{6 . 3 8 0 4}$ \\
Et Al. [5] & & & \\
\hline Proposed & $\mathbf{3 . 2 3 7 6}$ & $\mathbf{7 . 9 1 7 1}$ & $\mathbf{1 9 . 5 5 3}$ \\
\hline
\end{tabular}

Published By:

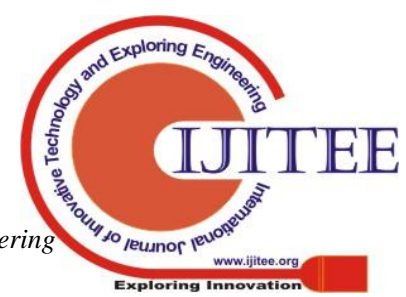




\section{Invisible Medical Image Watermarking using Edge Detection And Discrete Wavelet Transform Coefficients}
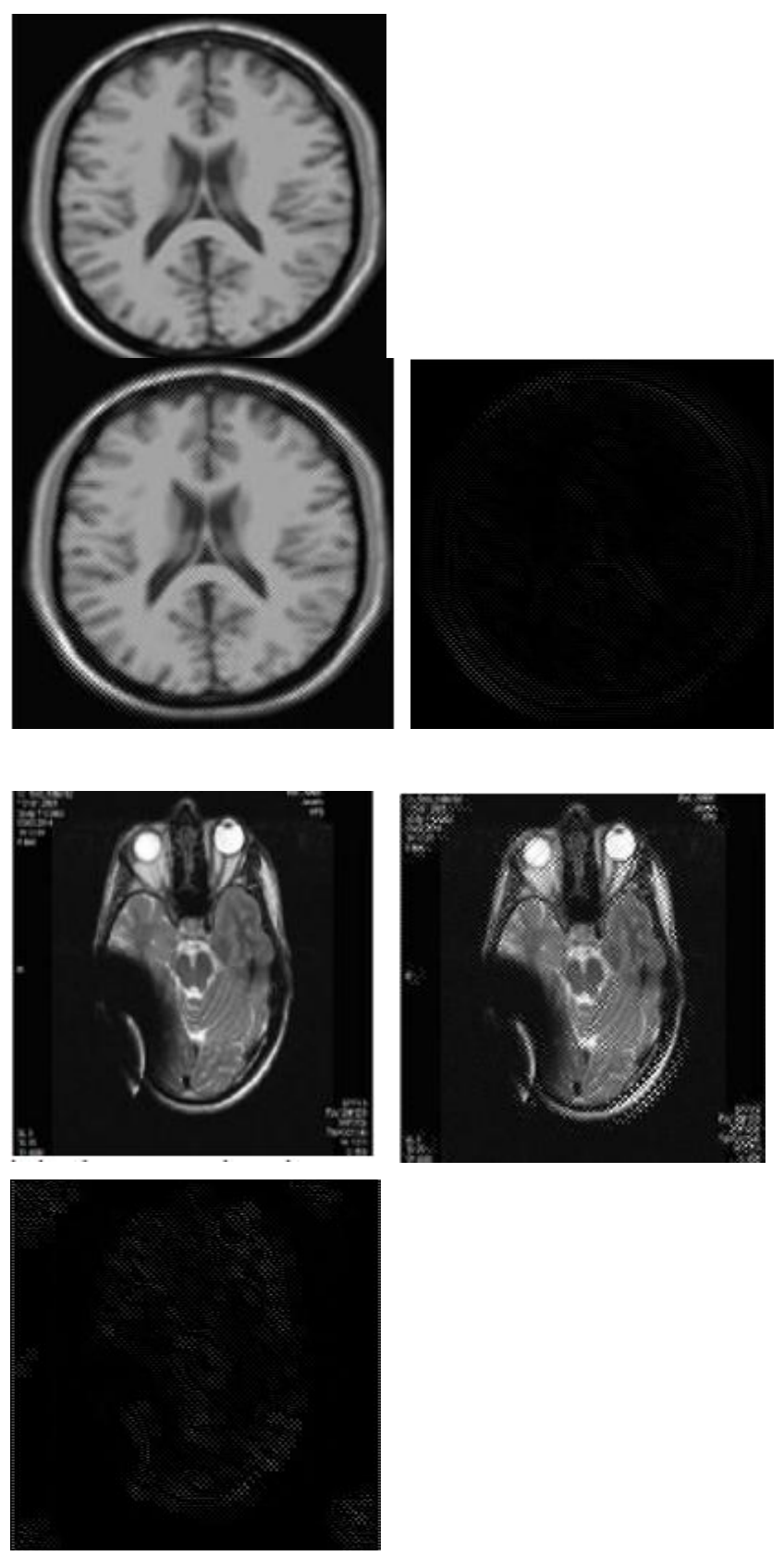

a)

B)

Figure 9. Watermarked image a) Original picture b) Watermarked picture c) difference $\mathbf{a}, \mathbf{b}$

\section{CONCLUSION}

In the manuscript a robust watermarking scheme is projected with edge detection with scaled dilation in 2-D DWT transform domain. Various medical images are tested and DWT based methods are implemented and compared in the current work. Paper presented the comparative results of the stage wise watermarking scheme and also compared with the method of Apeksha [5] Sobal edge detectors for wavelet based edge detectors are used with two level DWT decomposition and the comparative results are presented. It is found that wavelet based results with multi resolution are better and stable edge detectors perform equally for all kinds of medical images. The use of $2 \mathrm{X} 2$ dilation mask provides the better invisibility to the watermark. The watermark is added to $\mathrm{HH}$ sub and so the invisibility is better. But using dilation may degrade the invisibility of the watermark. Thus to improve the performance in this paper watermark is scaled using a scaling parameter. Scaling is only done on the coefficient difference and preserving the noise template improves the performance. In the future the adaptive scaling of the watermark can be implemented. In future the watermark detection can be implemented and the performance of the watermark detection under the various attacks can be evaluated. Performance of various wavelet filers may be evaluated in the future.

\section{REFERENCES}

1. John N. Ellinas, A Robust Wavelet-Based Watermarking Algorithm Using Edge Detection, IEEE Journal on image processing, pp. 197-208 2008.

2. Yingli Wang, Xue Bai,, Shuang Van "Digital Image Watermarking Based on Texture Block $<$ and Edge Detection in the Discrete Wavelet Domain" IEEE International Conference on Sensor Network Security Technology and Privacy Communication System (SNS \& PCS) pp. 170-174, 2013.

3. J. N. Ellinas, D. E. Manolakis, "A robust watermarking scheme based on edge detection and contrast sensitivity function," in VISAPP Proc. Int. Conf. Computer Vision Theory and Applications, Barcelona, 2007.

4. Narong Mettripun, "Robust Medical image watermarking based on DWT for Patient Identification", IEEE 13th international conference on electrical engineering/electronics, computer, telecommunications and information technology (ECTI-CON) July 2016.

5. Apeksha Tiwari, Virendra Singh "Digital Image Watermarking Using DWT and Shift Invariant Edge Detection "International Journal of Computer Technology and Electronics Engineering (IJCTEE) Volume 3, Issue 6, December 2013.

6. Nai-Kuei Chen, Chung-Yen Su, Che-Yang Shih, Yu-Tang Chen "Reversible Watermarking for Medical Images Using Histogram Shifting with Location Map Reduction", IEEE international Conference on Industrial Technology (ICIT) 2016.

7. I. J. Cox, J. Kilian, T. Leighton, and T. Shamoon, "Secure spread spectrum watermarking for multimedia," IEEE Trans. Image Processing, vol. 6, no. 12, pp. 1673-1687, Dec. 1997.

8. J. M. Zain and A. R. M. Fauzi, "Medical image watermarking with tamper detection and recovery", in Proceedings of the 28th IEEE EMBS Annual International Conference, pp.3270- 3273, 2006.

9. S. C. Liew and J. M. Zain, "Reversible medical image watermarking for tamper detection and recovery", 3 RD IEEE International Conference on Computer Science and Information Technology (ICCSIT), pp. 417-420, 2010.

10. Smita Agrawal, Manoj Kumar, “"'Reversible Data Hiding for Medical Images using Integer-to-Integer Wavelet Transform", In IEEE Students' Conference on Electrical, Electronics and Computer Science, (SCEECS) 2016.

11. R. Dugad, K. Ratakonda, and N. Ahuja, "A new wavelet-based scheme for watermarking images," in IEEE Proc. Int. Conf. Image Processing, USA, pp. 419-423, 1998.

12. J. R. Kim, and Y. S. Moon, "A robust wavelet-based digital watermarking using level-adaptive thresholding," in IEEE Proc. Int. Conf. Image Processing, Japan, 1999, pp. 226-230.

13. P. V. V. Kishore, S. R. C. Kishore, E. Kiran Kumar, K. V. V. Kumar, P. Aparna, "Medical image Watermarking with DWT-BAT algorithm", IEEE international conference on Signal Processing and Communication Engineering Systems (SPACES) 2015.

14. R. B. Wolfgang, C. I. Podilchuk, and E. J. Delp, "Perceptual watermarks for digital images and video," in SPIE Proc. Int. Conf. Security and watermarking of multimedia contents, USA, 1999, pp. 40-51.

15. R. J. Beattie, 1984, "Edge detection for semantically based early visua processing, "dissertation, Univ. Edinburgh, Edinburgh, U.K.

16. Vikas Tyagi "Data Hiding in Image using least significant bit with cryptography", International Journal of Advanced Research in Computer Science and Software Engineering, Vol. 2, Issue 4, April 2012.

17. S. Mallat, S. Zhong, 1992, "Characterization of signals from multistage edges,", IEEE Trans. Pattern Anal. Machine Intell., vol.14, no.7, pp. 710-732.

18. Xia Xiang-Gen, Boncelet, C.G., Arce, G.R., "A multiresolution watermark for digital images", in the proceedings of IEEE International Conference on Image Processing, Vol. 1, pp 549 - 551, Oct 1997.

\section{Published By:}

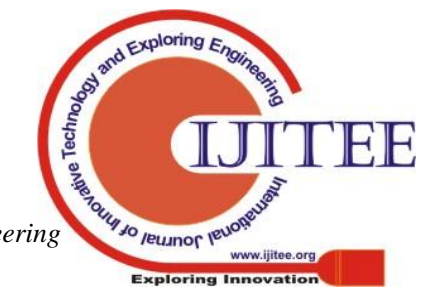


19. Tao, P \& Eskicioglu, "A Robust Multiple Watermarking Scheme in the Discrete Wavelet Transform Domain", in Symposium on Internet Multimedia Management Systems V, Philadelphia, PA, 2004.

\section{AUTHORS FROFILE}

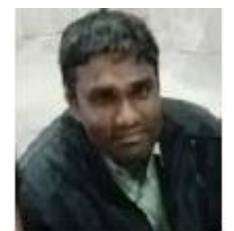

Ramanand Singh: has completed B.E. In Electronics Engineering in the 2003 LNCT bhopal, $\mathrm{M}$ Tech in microwave and milemetre from MANIT Bhopal INDIA in 2009, and pursuing Ph.D from AISECT University, Bhopal, India. Currently working as Associate Professor in the department of electronic and communication engineering at LNCT

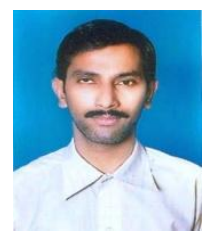

Paresh Rawat: has completed B.E. in Electronics Engineering in the 2000, $M$ Tech in Digital Communication from MANIT Bhopal INDIA in 2007, and Ph.D from MANIT INDIA, He is Member, IEEE and IETE. Currently working as Professor in the department of electronic and communication engineering at Sistech Bhopal, India. His main research areas are: Image and Video Processing He has been awarded as Bhaskars teacher facilitation award 2016 and best Faculty CII Tech Guru Award in 2017.

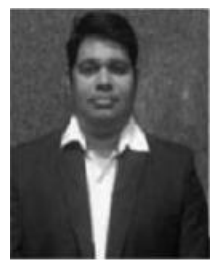

Dr. Piyush K. Shukla: Has received his Bachelor's degree in Electronics \& Communication Engineering, LNCT in 2001, Bhopal, M. Tech (Computer Science \& Engineering) in 2005 from SATI, Vidisha, Ph.D. (Computer Science \& Engineering) in 2013 from RGPV, Bhopal. M.P. India. He is a member of IACSIT He has published more than 15 papers in reputed International Journals and 10 papers in International Conferences. At present, he is working as an Assistant Prof. In Department of Computer Science \& Engineering, UIT-RGPV, Bhopal Since July 2007.

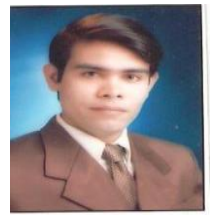

Dr. Prashant Kumar Shukla has joined Department of Computer Science and Engineering, JLU-School of Engineering \& Technology, Jagran Lakecity University, Bhopal (M.P.) in July, 2019. He is $\mathrm{PhD}$ in Computer Science and Engineering from Dr K. N. Modi University, Rajasthan. $\mathrm{He}$ is Master of Engineering from Rajiv Gandhi Proudyogiki Vishwavidyalaya, Bhopal. He is in research, teaching and industry for the past 18 years and working in the research areas like Machine Learning, Deep Learning, Computer Vision, Internet of Things (IOT) etc. He has applied for 14 patents, in which 13 patents has been published. He also has published and presented more than 13 research papers in various national and international SCI/Scopus/Indexed journals and conferences. He has published 02 Chapters in Scopus indexed edited book available in Google Books. 